and age. Am. J. Pub. Health 78 (1988) 1150-1155.

[3] Enstrom, J. E., Kanim, L. E., and Klein, M. A.: Vitamin $C$ intake and mortality among a sample of the United States population. Epidemiology 3 (1992) 194-202.

[4] Herrmann-Kunz, E., und Thamm, M.: Energie- und Nährstoffaufnahme in den neuen Bundesländern. In: Bellach, B.-M. (Hrsg). Die Gesundheit der Deutschen. Bd. 2. RKIHeft 15/1996, 89-100.

[5] Statistisches Jahrbuch 1995 für die Bundesrepublik Deutschland. Statistisches Bundesamt. Stuttgarr: Metzler-Poeschel Verlag 1995.

[6] Wiesner, G.: Ausgewählte Ergebnisse zur ambulanten Inanspruchnahme und ambulanten Morbidität. Z. ärztl. Fortbild. 79 (1985) 23-24.

[7] Bergmann, E.: Ambulante Inanspruchnahme I. In: Hoffmeister, H., und Bellach, B.-M.: Die Gesundheit der Deutschen. RKI-Heft 7/1995, 174-181.

[8] Bundesministerium für Gesundheit: Statistisches Taschenbuch Gesundheit. Baden-Baden: Nomos-Verlag 1996.

[9] Wiesner, G., Todzy-Wolff, I., und Hoffmeister, H.: Herzinfarkt. In: Hoffmeister, $H$. und Bellach, B.-M: Die Gesundheit der Deutschen. RKI-Heft 7/1995, 1 l-25.
[10] Busfield, J.: Men, women and madness. London: Macmillan press 1996.

[11] Wiesner, G.: Osteoporose - ein prioritäres Gesundheitsproblem mir vielen offenen Fragen. Bundesgesundhbl. 41, 6 (1998) 244-449.

[12] MacQueen, J. B.: Some Methods for Classification and Analysis of Multivariate Observations Proceedings of the Fifth Berkeley Symposium on Mathematical Statistics und Probability 1 (1967) 281-297.

[13] Harrigan, J. A.: Clustering Algorithms. New York: John Whey \& Sons 1975.

[14] Grimm, J., Kant, H., und Wiesner, G.: Multimorbidität. Forschungsbericht. Projekt Nr.: 19. Robert Koch-Institut, Berlin 1997.

[15] Waldron, I.: Sex differences in illness incidence, prognosis and mortality: issues and evidence. Soc. Sci. Med. 17 (1983) 1107-1123.

[16] Lang, E., Arnold, K., und Kupfer, P.: Frauen werden älter - biologische, medizinische und soziologische Ursachen. Z. Gerontol. 27 (1994) $10-15$.

[17] Casper, W., Wiesner, G., und Bergmann, K.: Mortalität in Deutschland - unter besonderer Berücksichtigung der Entwicklung in den alten und neuen Bundesländern. RKI-Heft 10/1995.

[18] Kühne, K., und Brahms, O.: Die Prognose der essentiellen Hypertonie. Darmstadt: Steinkopf 1964.
[19] Jahnecke, J.: Risikofaktor Hypertonie. Hochdruckfibel für die ärztliche Praxis. Studienreihe Boehringer Mannheim. Mannheim: Mannheimer Morgen Großdruckerei und Verlag GmbH 1974.

[20] Schwarzer, R.: Psychologie des Gesundheirsverhaltens. Göttingen: Hogrefe-Verlag 1996.

[21] Barnett, R. C., and Marshall, N. L.: Men, family-role, job-role quality and physical health. Health Psychology 122 (1993) 48-55.

[22] Hibbard, J. H., and Pope, C. R.: The quality of social rcles as predictors of morbidity and mortality. Soc. Sci. Med. 36 (1993) 217-222.

[23] Verbrugge, L. M.: The twain meet: Empirical explanations of sex differences in health and mortality. J. Health Soc. Behv. 30 (1989) 282-304.

[24] Klotz, Th., Hurrelmann, K., und Eickenberg, H.-U.: Der frühe Tod des starken Geschlechts. Deutsches Ärzteblatt 95 (1998) $362-366$.

Verfasser:

Dr. Jürgen Grimm, Edelgard Bittner und Doz. Dr. Dr. Gerd Wiesner, Robert Koch-Institut, Berlin

Korrespondenzanschrift:

Doz. Dr. Dr. G. Wiesner, Robert Koch-Institut, Postfach 650280, 13302 Berlin

\title{
Zum geschlechtsdifferenten Prozeß der Lebensverlängerung in Deutschland
}

\author{
Von G. Wiesner, E. Bittner und I. Todzy-Wolff
}

\section{Zusammenfassung}

Der Lebensverlängerungsprozeß hat sich in diesem Jahrhundert in Deutschland fortgesetzt, wobei die weibliche Bevölkerung einen höheren Zugewinn an Lebenserwartung gegenüber der männlichen Bevölkerung realisieren konnte. Für die Zeitintervalle 1949/51 bis $1960 / 62,1960 / 62$ bis $1970 / 72$, $1970 / 72$ bis $1981 / 83$ und $1981 / 83$ bis 1993/95 wurden für die männliche und weibliche Bevölkerung der Bundesrepublik Deutschland (früheres Bundesgebiet) die relativen Veränderungen der altersspezifischen Überlebenswahrscheinlichkeiten errechnet. Während der Lebensverlängerungsprozeß der weiblichen Bevölkerung über alle Zeitintervalle nahezu kontinuierlich voranschreitet, waren bei der männlichen Bevölkerung in definierten Altersbereichen erhebliche Schwankungen zu beobachten. Der Lebensverlängerungs- prozeß wird bei beiden Geschlechtern zunehmend im höheren Lebensalter wirksam. $\mathrm{Da}$ in den aufeinanderfolgenden Geburtsjahrkohorten die genetisch/biologische Variabilität weitgehend konstant reproduziert wird, ist die geschlechtsdifferente Entwicklung der Lebenserwartung sozialer Natur. Der höhere säkulare Lebenserwartungszugewinn der weiblichen Bevölkerung ist Ausdruck einer höheren Partizipation an der sozialen Aufwärtsentwicklung.

\section{Einführung}

Die Sterbetafeln der männlichen und weiblichen Bevölkerung der Bundesrepublik Deutschland weisen für den Zeitraum der Jahre 1993/95 eine mittlere Lebenserwartung der Männer von 72,99 Jahren gegenüber 79,49 bei den Frauen aus. Männliche Lebendgeborene sterben also, den Sterblichkeitsverhältnissen des Zeitraumes 1993/95 entsprechend, durchschnittlich 6,5 Jahre früher als die gleichzeitig lebendgeborenen Mädchen.

Daß die Sterblichkeit und die Lebenserwartung Unterschiede aufweist, ist keine neue Erkenntnis. Die Lebenserwartung der Frauen war nach den offiziellen Sterbetafeln in den letzten 125 Jahren in Deutschland in allen Altersstufen höher als die der Männer. Die männliche Bevölkerung hat demnach ein generell höheres Sterberisiko als die weibliche Bevölkerung. Die Geschlechtsdifferenzen in der Lebenserwartung zeichnen sich vor dem Hintergrund einer stetigen Zunahme der Lebenserwartung bei beiden Geschlechtern ab. Es stellt sich hier die Frage, ob die männliche Bevölkerung eine verminderte Dynamik des Lebensver- 
längerungsprozesses aufweist und wie sich die Geschlechtsdifferenzen der Lebenserwartungswerte in der Bundesrepublik Deutschland entwickelt haben.

Für die Entwicklung eines so komplexen Datums wie der Lebenserwartung zeichnet nicht ein einzelner Umstand verantwortlich, sondern vielmehr übergreifende systematische Einflußbereiche, wie etwa soziale und ökonomische Bedingungen, Lebensqualität, allgemeine Lebenszufriedenheit, gesundheitsbezogene Handlungs- und Verhaltensweisen, medizinische Versorgung etc. Somit ist der Lebensverlängerungsprozeß der allgemeine Ausdruck der sozialen Entwicklung menschlicher Kulturen, und steigende Lebenserwartungswerte sind grundsätzlich mit besseren gesundheitlichen Verhältnissen gleichzusetzen. Nur im Falle einer fortdauernden sozialen Stagnation können durch isolierte und selektive medizinische Versorgungsprogramme die Sterblichkeiten weiter sinken. An die Stelle der sozialen Entwicklung als Hauptursache der Lebensverlängerung tritt das System der medizinischen Versorgung. Diese „Entkopplung" kann wegen der komplexen Folgen der Lebensverlängerung nun ihrerseits zu einer umfassenden Last für die weitere soziale Entwicklung werden. Diese Situation ist für die Bundesrepublik Deutschland nicht auszumachen. Ungeklärt bleibt aber das Problem, ob die niedrigere Lebenserwartung der männlichen Bevölkerung Ausdruck einer geringeren Partizipation an der sozialen Aufwärtsentwicklung ist.

\section{Material und Methodik}

Ausgangsmaterial sind die Sterbetafeln des deutschen Reiches (jeweiliger Gebietsstand) 1901/1910, 1932/1934 und die der Bundesrepublik Deutschland (früheres Bundesgebiet) 1949/1951 (ohne Saarland und Berlin-West), 1960/1962,1970/1972, 1981/1983, 1993/ 1995. Die Sterbetafel ist ein Modell zur Berechnung der Maße der Lebensdauer. Neben der Sterblichkeit bzw. der Lebensdauer beeinflussen auch Geburtlichkeit sowie $\mathrm{Zu}$ - und Abwanderung die Alters- und Geschlechtsstruktur der Bevölkerung. Die völlige Ausschaltung aller die Vergleichbarkeit beeinträchtigenden Faktoren ist nur mit der Sterbetafelmethode möglich. Die Sterbetafel beruht auf dem theoretischen Konzept einer stationären Bevölkerung. Eine stationäre Bevölkerung ist durch das Gleichgewicht von Geburtlichkeit und
Sterblichkeit gekennzeichnet. Bei der Berechnung einer Sterbetafel wird eine fiktive, konstante Menge Lebendgeborener (Kohorte) mit dem Anfangsbestand von 100000 den aiters- und geschlechtsspezifischen Sterbewahrscheinlichkeiten $q_{x}$ der realen Bevölkerung ausgesetzt. Dadurch entsteht wiederum eine fiktive Bevölkerung (Sterbetafelbevölkerung), die im Umfang und Struktur ausschließlich von den Sterblichkeitsverhältnissen der zugrundeliegenden realen Bevölkerung bestimmt wird und damit deren exakte Vergleichbarkeit in der Zeit garantiert. Die Sterbetafelmethode muß als die Methode der Wahl zur Darstellung von Differenzen des Sterberisikos der männlichen und weiblichen Bevölkerung angesehen werden. Der Ausgangspunkt für das Berechnen von Sterbetafeln sind demnach die altersspezifischen Sterbewahrscheinlichkeiten $\left(\mathrm{q}_{\mathrm{x}}\right)$ der realen Bevölkerung nach Alter $» x \ll$. Die $»$ mittlere Lebenserwartung " $\left(e_{0}\right)$ ist die spezifische Interpretation der Gesamtheit der aktuell zu schätzenden Überlebenswahrscheinlichkeiten $\left(p_{x}\right)$ aller einzelnen Altersjahre x bzw. der dazu komplementären altersspezifischen Sterbewahrscheinlichkeiten $\left(q_{x}\right)$ für den Übergang von einem Alter $x$ zu dem Alter $x+1$. Die $\gg$ ferneren Lebenserwartungswerte $e_{\mathrm{x}}$ interpretieren die Gesamtheir der Überlebens- bzw. Sterbewahrscheinlichkeiten der Altersjahre y, die gleich oder größer $x$ sind. Die Beziehungen in der Sterbetafel lassen sich durch folgende Symbole und Gleichungen ausdrücken [1-3]:

1. $\mathrm{p}(0, \mathrm{x})=$ Wahrscheinlichkeit, das Alter $x$ zu überleben mit $\mathrm{p}(0,0)=1\left(\right.$ Tafelsymbolik $\left.\approx 1_{\mathrm{x}} / 1_{0}\right)$ $\mathrm{l}_{\mathrm{x}}=$ Überlebende im Alter $\mathrm{x}$ $\mathrm{l}_{\mathrm{o}}=$ Überlebende im Alter 0

2. $k(0, x)=$ Wahrscheinlichkeir, im Altersintervall [ 0 bis $x$ ] zu sterben, $d$. h. $\mathrm{k}(0, \mathrm{x})=1-\mathrm{p}(0, \mathrm{x})$, (kumulierte $\mathrm{Ha}$ sardrate $), k(0,101)=1$,

3. $r(x)=\partial / \partial x(k(0, x))=-\partial / \partial x(p(0, x))$, dabei sind die $\partial / \partial \mathrm{x}$ Operatoren für (Tafelsymbolik $\approx \mathrm{d}_{\mathrm{x}} / \mathrm{l}_{\mathrm{o}}$ ) die Ableitung nach $\mathrm{x}$ (Differentialquotienten) und $r(x)$ ist die Wahrscheinlichkeit, als 0jähriger im Alter $x$ zu sterben.

$\mathrm{d}_{\mathrm{x}}=$ Gestorbene im Alter $\mathrm{x}$ bis unter $\mathrm{x}+1$.

Die Funktion $r(x)$ ist eine Wahr scheinlichkeitsdichte, so daß das Integral über alle Alterswerte $\mathrm{x}$ den Wert 1 ergibt. In der Sterbetafel gilt:

$$
\sum \mathrm{d}_{\mathrm{x}}=\mathrm{l}_{\mathrm{o}}=100000 \text {. }
$$

4. $q_{x}=r(x) / p(0, x)=$ Wahrscheinlichkeit, als xjähriger zu sterben (altersspezifische Sterbewahrscheinlichkeit - Tafelsymbolik $\approx q_{x}$,

5. $p_{x}=1-q_{x}=$ Wahrscheinlichkeit, den Alterspunkt $x$ zu überleben (Überlebenswahrscheinlichkeit - Tafelsymbolik $\approx \mathrm{p}_{\mathrm{x}}$ ),

6. $e_{0}=\int_{0}^{101} x \cdot r(x) d x$, die mittlere Lebenserwartung, wenn über den ganzen Altersbereich integriert wird. Integriert man das vorstehende Integral unter Anwendung der Produktregel und verwendet die Beziehungen $r(x)=$ $\partial / \partial \mathrm{x} \mathrm{k}(0, \mathrm{x})$ und $1-\mathrm{k}(0, \mathrm{x})=\mathrm{p}(0, \mathrm{x})$, so ergibt sich $e_{0}=\int_{0}^{101} p(0, x) d x$, integriert über den möglichen Altersbereich bis 101 Jahre.

In der Sterbetafel ergibt sich damit:

7. $e_{0} \approx\left(\sum l_{x} / l_{o}\right)$. Der Quotient der Sterbetafelwerte $l_{x} / l_{o}$ entspricht der Wahrscheinlichkeit $\mathrm{p}(0, \mathrm{x})$ und ist das fortlaufende Produkt der Überlebenswahrscheinlichkeiten $\mathrm{p}_{\mathrm{x}}$.

Der Lebensverlängerungsprozeß zeigt zwischen Ost- und Westdeutschland nach 1945 eine große Verschiedenheit auf [1], so daß in der vorliegenden Arbeit nur auf die Sterbetafeln des früheren Bundesgebietes zurückgegriffen wird. Die relative Veränderung der Überlebenswahrscheinlichkeiten ist ein beliebtes Maß, um den Einfluß von Mortalitätsveränderungen auf die Alterszusammensetzung des jeweiligen Geschlechts zu untersuchen [4-6]. Indem man die relativen Veränderungen der Überlebenswahrscheinlichkeiten in

Tabelle 1: Mittlere Lebenserwartungen für die deutsche Bevölkerung*

\begin{tabular}{llll}
\hline Jahr & männlich & weiblich & $\begin{array}{l}\text { Differenz } \\
\text { in Jahren } \\
\text { w/m }\end{array}$ \\
\hline $1901 / 10$ & 44,82 & 48,33 & 3,51 \\
$1924 / 26$ & 55,97 & 58,82 & 2,85 \\
$1932 / 34$ & 59,86 & 62,81 & 2,95 \\
$1949 / 51$ & 64,56 & 68,48 & 3,92 \\
$1960 / 62$ & 66,86 & 72,39 & 5,53 \\
$1970 / 72$ & 67,41 & 73,83 & 6,42 \\
$1981 / 83$ & 70,46 & 77,09 & 6,63 \\
$1993 / 95$ & 73,53 & 79,81 & 6,28 \\
\hline
\end{tabular}

bis 1932/34 Reichsyebier, jewciliger Gebietsstand; 1949/51 früheres Bundesgebiet ohne Saarland und Berlin, bis 1993/95 früheres Bundesgebiet 
Deutschland beispielsweise für die Epoche von 1901/10 bis 1993/95 und $1901 / 10$ bis 1960/62 nebeneinander stellt, kann man jene Altersintervalle der männlichen und der weiblichen Bevölkerung identifizieren und untersuchen, in denen größere Veränderungen stattgefunden haben.

\section{Ergebnisse}

Im 20. Jahrhundert entwickelte sich die mittlere Lebenserwartung in Deutschland wie folgt (Tab. 1). Der Tabelle 1 ist $\mathrm{zu}$ entnehmen, daß der Lebensverlängerungsprozeß sich in diesem Jahrhundert in Deutschland bis zur Gegenwart fortgesetzt hat: $\operatorname{der} \mathrm{Zu}-$ gewinn an Lebenserwartung beträgt 28,71 Jahre bei der männlichen Bevölkerung und 31,48 Jahre bei der weiblichen Bevölkerung. Demnach konnte die weibliche Bevölkerung bei schon höheren Ausgangswerten der mittleren Lebenserwartung am Anfang dieses Jahrhunderts einen um 2,77 Lebensjahre höheren Zugewinn der Lebenserwartung gegenüber der männlichen Bevölkerung realisieren. Am Ausgang dieses Jahrhunderts (Abb. 1) hat die weibliche Bevölkerung bezogen auf die Sterbetafel 1993/95 für das frühere Bundesgebiet eine um 6,28 Jahren höhere Lebenserwartung. Am Zugewinn der Lebenserwartung sind alle Altersklassen beteiligt.

Aus der Abbildung 2 wird aber schon ersichtlich, daß der Beitrag der einzelnen Altersklassen an der Erhöhung der Lebenserwartung unterschiedlich ausfällt. Im ersten Drittel dieses Jahrhunderts (1901/1910 bis 1932/1934) dominieren bei beiden Geschlechtern die Null- bis unter Einjährigen. Im letzten Drittel dieses Jahrhunderts sind die $\mathrm{Zu}$ gewinne in den Altersklassen der über 60jährigen am größten. Bei genauer Betrachtung der Abbildung 2 wird offenkundig, daß im ersten Drittel des Jahrhunderts die männliche Bevölkerung eine stärkere Steigerung der Lebenserwartungswerte aufweist als die weibliche Bevölkerung.

Nach 1924/1926 (siehe Tab. 1) hat die weibliche Bevölkerung bis Anfang der 80er Jahre stets eine höhere Zunahme der Lebenserwartung zu verzeichnen; in den 90er Jahren hat sich die Differenz zwischen den männlichen und weiblichen Lebenserwartungswerten erneut etwas vermindert.

Die Abbildung 3 zeigt die relative Veränderung der Überlebenswahrscheinlichkeiten in Deutschland in diesem

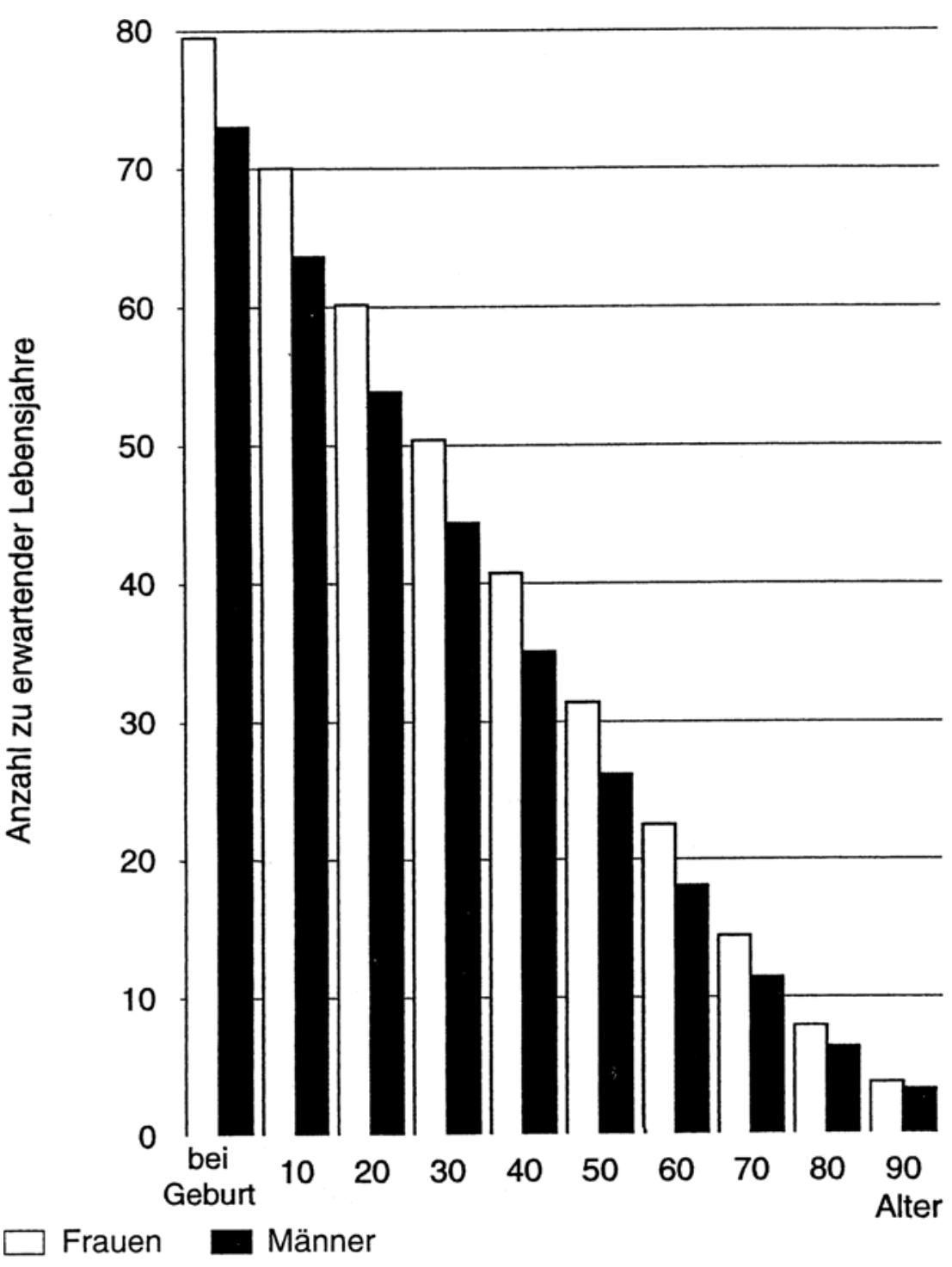

Datenquelle: StBA, Statistisches Jahrbuch 1996

Abbildung 1: Lebenserwartung nach Alter und Geschlecht, Deutschland 1993/95.

Jahrhundert. Indem man die prozentualen Veränderungen der Überlebenswahrscheinlichkeiten der einzelnen Altersjahre für die Periode von 1901/1910 bis 1993/1995 und der entsprechenden Werte für die Periode von 1901/10 bis 1960/62 erfaßt, kann man deutlich erkennen, daß die Veränderungen in den Altersklassen der unter Fünfjährigen und der über 65jährigen am größten sind. In den Altersklassen dazwischen verbessern sich die Überlebenschancen nur geringfügig. Eingebettet in den säkularen Lebensverlängerungsprozeß müssen sich in der letzten Periode (1960/62 bis 1993/95) neue Veränderungen hauptsächlich im höheren Altersbereich ereignet haben. Hier haben signifi- kante Mortalitätsreduktionen stattgefunden, wodurch sich die Überlebenszeiten der Altenbevölkerung erheblich verlängert haben. Der Verlauf der Kurven zeigt, daß die Verbesserung der Überlebenschancen im höheren Lebensalter bei den Frauen ausgeprägter ist als bei den Männern.

In den Tabellen 2 und 3 sind detailliert die relativen Veränderungen der Überlebenswahrscheinlichkeiten für die Bundesrepublik Deutschland (früheres Bundesgebiet) für die Zeitintervalle $1949 / 51$ bis $1960 / 62,1960 / 62$ bis $1970 / 1972,1970 / 72$ bis $1981 / 83$ und $1981 / 83$ bis $1993 / 95$ aufgeführt. Die Tabellen belegen, daß der Lebensveriänge- 
rungsprozeß der weiblichen Bevölkerung über alle Zeitintervalle nahezu kontinuierlich voranschreitet, während bei der männlichen Bevölkerung erhebliche Schwankungen zu beobachten waren. Die Männer, die 1960/62 älter als 55 Jahre waren, zeigen gegenüber denen der Jahre 1949/51 Verluste an Lebenserwartung. Die hier betroffenen Kohorten (ab 1906 bis 1881) sind auch im folgenden Zeitintervall $1960 / 62$ bis $1970 / 72$ auffällig. 1970/72 fallen noch weitere Kohorten durch Negativwerte im Altersbereich zehn bis 20 Jahre (z. B. im Zusammenhang mit der zunehmenden Motorisierung ) und 35 bis 50 Jahre (z. B. infolge kriegsbedingter Langzeitschäden) gegenüber 1960/62 auf. Für die weibliche Bevölkerung der Bundesrepublik wird - abgesehen von geringfügigen Störungen in der Periode 1960/62 bis $1970 / 72$ - eine monotone Verbesserung der Überlebensmöglichkeiten seit 1949/51 deutlich, wobei insbesondere seit 1960/62 im Altersbereich ab 55 Jahre deutliche Gewinne an Lebenserwartung zu beobachten sind. Kohorteneffekte wie bei den Männern treten nicht auf (Abb. 4).

Die Entwicklungen des Lebensverlängerungsprozesses waren zwischen der männlichen und weiblichen Bevölkerung in der Periode 1949/51 bis 1970/72 in definierten Altersbereichen divergent; in der folgenden Periode 1970/72 bis 1993/95 wird eine unverminderte Dynamik des Lebensverlängerungsprozesses bei beiden Geschlechtern erkennbar. Die relativen Veränderungen der Überlebenswahrscheinlichkeiten besagen dabei, daß insbesondere in hohen und höchsten Altersbereichen Lebenszeit in erheblichem Umfang gewonnen wird. Die Werte in den Tabellen 2 und 3 lassen deutlich erkennen, daß der $\mathrm{Al}$ tersbereich der Säuglinge, Kinder und Jugendlichen für die Entwicklung der
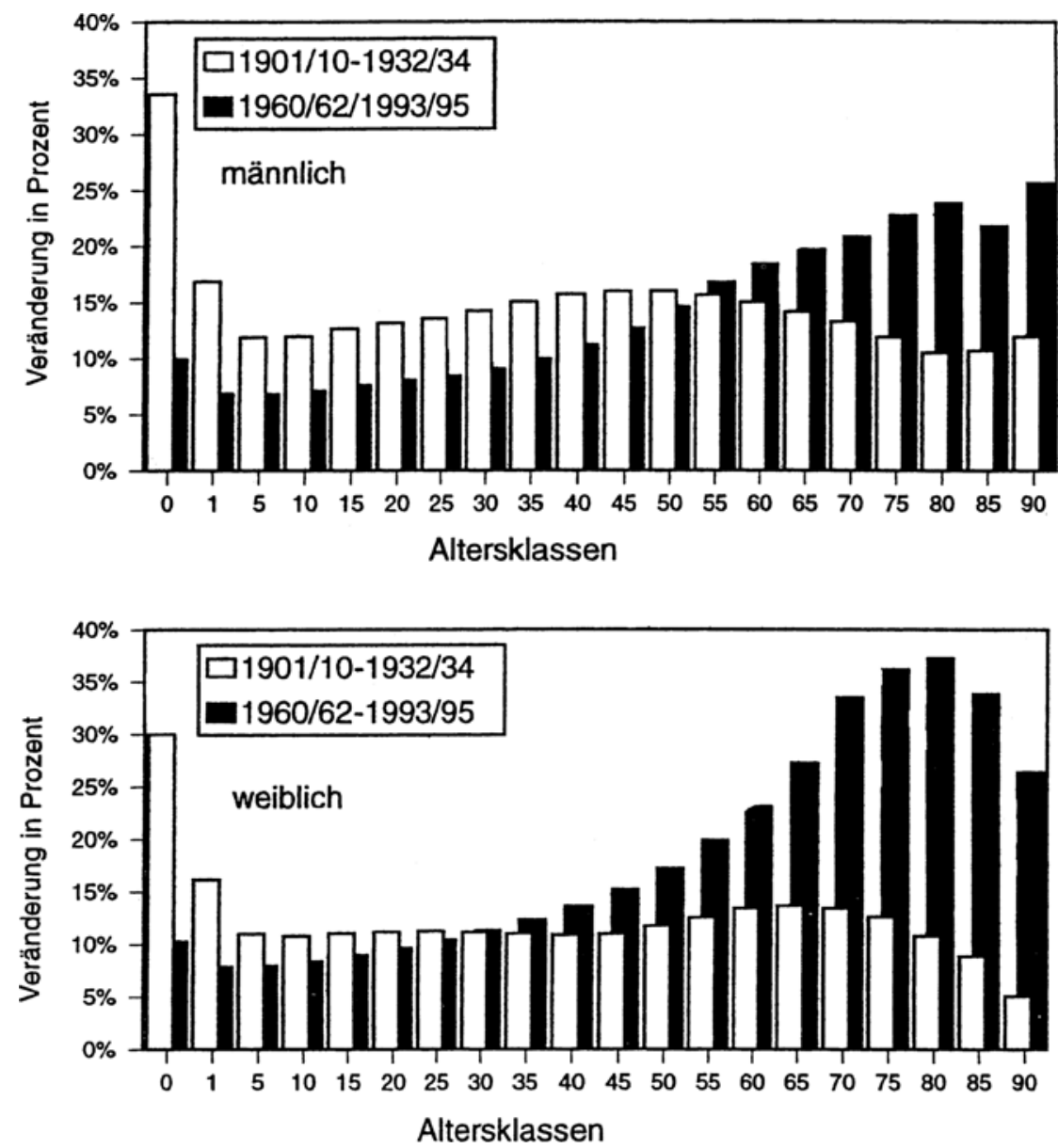

Quelle: Berechnungen nach Daten des Statistischen Bundesamtes; 1901/10-1932/34 Deutsches Reich, 1960/62-1993/95 früheres Bundesgebiet

Abbildung 2: Prozentuale Zunahme der Lebenserwartung nach Alter in den Perioden $1901 / 10$ bis $1932 / 34$ und $1960 / 62$ bis $1993 / 95$.
Lebenserwartungen im Zeitintervall $1981 / 83$ bis 1993/95 nahezu bedeutungslos geworden ist. Der Lebensverlängerungsprozeß wird also zunehmend im höheren Lebensalter wirksam, in dem chronisch-degenerative Krankheiten das Sterbegeschehen dominieren.

\section{Diskussion}

Eine höhere Lebenserwartung der weiblichen Bevölkerung findet sich heute in allen Regionen der Welt, soweit überhaupt Daten zur Lebenserwartung vorliegen. In Deutschland und in den entwickelten europäischen Ländern werden erst seit etwa 300 Jahren höhere Lcbenserwartungswerte der weiblichen Bevölkerung beobachtet. Vor diesem Zeitraum war die weibliche Lebenserwartung infolge einer enorm hohen Sterblichkeit in der Fertilitätsperiode geringer als die männliche. Zwischen dem Ende des zehnten bis Mitte des zwölften Jahrhunderts überlebten in Deutschland vermutlich nur etwa $20 \%$ der in die Fertilitätsperiode eintretenden Frauen das 40. Lebensjahr. In einigen Entwicklungsländern (soweit überhaupt Daten zur Lebenserwartung vorliegen) fanden sich noch bis in die 60er/70er Jahre niedrigere Lebenserwartungswerte der weiblichen Bevölkerung gegenüber der männlichen Bevölkerung.

Die Geschlechtsdifferenz in der Lebenserwartung hat sich in Deutschland vom Anfang dieses Jahrhunderts bis Anfang der 80 er Jahre ständig zuungunsten des Mannes vergrößert. Mehr als die Hälfte der weiblichen Bevölkerung wird gegenwärtig 80 Jahre alt und älter, während es bei der männlichen Bevölkerung nur etwas mehr als ein Drittel ist.

Zur Erklärung der höheren Lebenserwartung der Frauen sind viele Fakten heranzuziehen, deren tiefere ursächliche Zusammenhänge noch längst nicht alle bekannt sind. Die Ungleichheit in der Lebenserwartung zwischen Männern und Frauen ist die Folge ihrer genetisch/biologischen Variabilität und der Modifikabilität in Abhängigkeit von den sozialen Existenzbedingungen. In den aufeinanderfolgenden Geburtsjahrkohorten wird die genetisch/biologische Variabilität weitgehend konstant reproduziert, während sich die sozialen Existenzbedingungen systematisch verändern. Genetische Faktoren, die einen bestimmten Beitrag zur geschlechtsdifferenten Sterblichkeit bzw. Lebenserwartung leisten, bleiben demnach in der Zeit relativ stabil. 

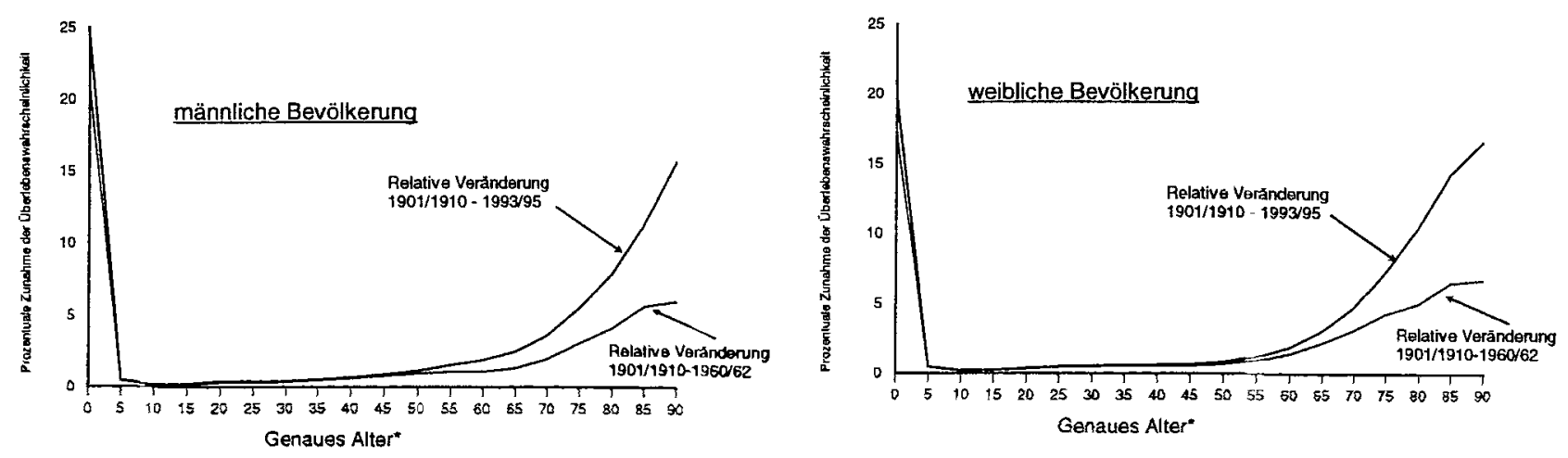

* Es beziehen sich: Das Alter 0 auf den Zeitpunkt der Geburt, die anderen Altersangaben auf den Zeitpunkt, an dem jemand genau x Jahre alt geworden ist.
* 1901/1910 Deutsches Reich, 1960/1962 und 1993/1995 früheres Bundesgebiet

Abbildung 3: Prozentuale Veränderungen der Überlebenswahrscheinlichkeiten der deutschen Bevölkerung nach Altersjahren für die Epoche $1901 / 10$ bis $1960 / 62$ und $1901 / 10$ bis $1993 / 95^{* \%}$.

Der Versuch einer Quantifizierung der durch Umwelteinflüsse modifizierten Anteile genetischer Faktoren auf die Sterblichkeit stößt auf unüberbrückbare Schwierigkeiten. Gegenwärtig besteht in der Genomforschung noch das Unvermögen, das Zusammenspiel der Gene untereinander sowie mit der Umwelt zu verstehen. So werden sich beispielsweise die hohen Erwartungen bei der Inauguration des Humangenomprojekts in absehbarer Zeit kaum erfüllen. Eine genetische Anomalie richtig zu bewerten, stößt sogar bei monogenen Krankheiten auf diffizile Probleme (es besteht eine gewaltige Komplexität der Genome, die untereinander vernetzt sind und in Form von Regelkreisen ineinandergreifen). Als Beleg dafür gilt die Mukoviszidose. So läßt sich bisher kein Zusammenhang zwischen der Art der Veränderung in dem als CFTR bezeichneten Gen und der Erbkrankheit erkennen, obgleich man schon mehr als siebenhundert Mutationen auf dem Gen identifiziert hat.

Für die genetische Hypothese spricht, daß bereits in der intrauterinen Entwicklungsphase sowie in dem sich unmittelbar anschließenden Säuglingsund Kleinkindalter das männliche Geschlecht eine höhere Sterblichkeit aufweist. Schärzungen des primären Geschlechtsverhältnisses zum Implantationszeitpunkt beim Menschen liegen bei Werten von 140 bis 200 angelegten männlichen Früchten gegenüber 100 weiblichen Früchten. Weder der Mecha- nismus der überzähligen Anlage männlicher Früchte, noch die Ursachen der höheren fötalen Sterblichkeit des männlichen Geschlechts sind befriedigend geklärt. Immungenetische Reaktionen zwischen mütterlichem und kindlichem Organismus als Ursache der höheren Sterblichkeit männlicher Föten sind zu vermuten.

Eine Reihe von Autoren macht vor allem die Einflüsse der geschlechtsgebundenen unterschiedlichen »Hormonlagen «, die Blutfette und deren Metabolismen (insbesondere der Lipoproteinmetabolismus), den Kalzium- und Eisenstoffwechsel sowie des Hämatokrits in Abhängigkeit zu den Geschlechtshormonen verantwortlich [8-11]. Auch die rezessiven Erbkrankheiten gehen bei Männern in einem relativ kleinen Prozentsatz in die Gesamtsterblichkeit ein $[12,13]$. Nach neurobiochemischen Befunden muß auch an Impulskontrollstörungen im serotonergen System gedacht werden, wobei das männliche Geschlecht aufgrund seiner genetischen Disposition vermutlich vermehrt eine Serotonin-Stoffwechselstörung aufweist. Dies kann zu einer erhöhten Auto- und Fremdaggression führen [14]. Geschlechtsunterschiede im Risikoverhalten ist in sämtlichen Kulturen zu finden [15]. Die Sterblichkeit infolge von Infektionskrankheiten ist nach internationalen Sterblichkeitsvergleichen bei männlichen Säuglingen sowie für Männer über 40 konsistent höher als für Frauen. Es wird vermutet, daß geneti- sche Fakroren für eine höhere Empfindlichkeit des männlichen Geschlechts gegenüber Infektionen eine Rolle spielen [13]. Dabei wird eine Abhängigkeit von der X-Chromosomen verbundenen Autoimmunproduktion vermutet. Frauen hätten wegen der doppelt vorhandenen $\mathrm{X}$-Chromosomen eine erhöhte Autoimmunproduktion. X-chromosomverbundene immunregulatorische Gene wären dann die Ursache für eine größere Resistenz der Frauen gegenüber Infektionskrankheiten. Diese These müßte auch für andere Krankheiten zutreffend sein. Einen wesentlichen Einfluß auf die geschlechtsdifferente Lebenserwartung haben Herz-Kreislauf-Erkrankungen (insbesondere Herzinfarkt), Krebserkrankungen (z. B. Lungenkrebs), Krankheiten der Atmungsorgane (Pneumonie, Grippe, Asthma bronchiale), Krankheiten der Verdauungsorgane (insbesondere Leberzirrhose), Todesfälle nach Unfällen, Suiziden und anderen Gewalteinwirkungen [16].

Für den Komplex »Verhalten und Umwelt« als sozialwissenschaftlicher Erklärungsansatz sprechen die Ergebnisse einer Reihe epidemiologischer Studien: Rauchen, Alkohol, Drogen oder bestimmte Ernährungsbestandteile werden für das differente Sterberisiko verantwortlich gemacht. Die geschlechtsdifferenten Erziehungsmuster und Verhaltensstile werden dabei durch die soziale Umwelt vermitteit. Auch die $» s o-$ ziale Lage« wird eng mit der Lebenser- 
Tabelle 2: Überlebenswahrscheinlichkeiten nach Altersjahren und prozentuale Veränderungen, männliche Bevölkerung; Bundesrepublik Deutschland (früheres Bundesgebiet) 1949/51, 1960/62, 1970/72, 1981/83, 1993/95

männliche Bevölkerung

Voll-

Wahrscheinlichkeit,

Prozentuale Veränderungen

endetes das erreichte Altersjahr zu überleben

Altersjahr x

\begin{tabular}{llllll|llll}
\hline & $1949 / 51$ & $1960 / 62$ & $1970 / 72$ & $1981 / 83$ & $1993 / 95$ & $1949 / 51-1960 / 62$ & $1960 / 62-1970 / 72$ & $1970 / 72-1981 / 83$ & $1981 / 83-1993 / 95$ \\
\hline 0 & 0,93823 & 0,96467 & 0,974 & 0,98787 & 0,99384 & 2,82 & 0,97 & 1,42 & 0,60 \\
1 & 0,99584 & 0,99769 & 0,99845 & 0,99908 & 0,9995 & 0,19 & 0,08 & 0,06 & 0,04 \\
2 & 0,99754 & 0,9986 & 0,999 & 0,99943 & 0,99962 & 0,11 & 0,04 & 0,04 & 0,02 \\
5 & 0,99879 & 0,9992 & 0,99927 & 0,9996 & 0,9998 & 0,04 & 0,01 & 0,03 & 0,02 \\
10 & 0,9993 & 0,99955 & 0,99953 & 0,99975 & 0,99987 & 0,03 & $-0,002$ & 0,02 & 0,01 \\
15 & 0,99896 & 0,99925 & 0,99921 & 0,99951 & 0,99966 & 0,03 & $-0,004$ & 0,03 & 0,02 \\
20 & 0,99812 & 0,99815 & 0,998 & 0,99854 & 0,99903 & 0,003 & $-0,02$ & 0,05 & 0,05 \\
25 & 0,99777 & 0,99831 & 0,99839 & 0,99879 & 0,99907 & 0,05 & 0,01 & 0,04 & 0,03 \\
30 & 0,99772 & 0,9983 & 0,9983 & 0,99869 & 0,9989 & 0,06 & 0,0 & 0,04 & 0,02 \\
35 & 0,99724 & 0,99791 & 0,9979 & 0,99839 & 0,99854 & 0,07 & $-0,001$ & 0,05 & 0,02 \\
40 & 0,99648 & 0,99705 & 0,9968 & 0,99736 & 0,99777 & 0,06 & $-0,03$ & 0,06 & 0,04 \\
45 & 0,99484 & 0,99557 & 0,99525 & 0,99579 & 0,99663 & 0,07 & $-0,03$ & 0,05 & 0,08 \\
50 & 0,9915 & 0,99261 & 0,99229 & 0,99276 & 0,99443 & 0,11 & $-0,03$ & 0,05 & 0,17 \\
55 & 0,98725 & 0,98703 & 0,98794 & 0,98905 & 0,99152 & $-0,02$ & 0,09 & 0,11 & 0,25 \\
60 & 0,98109 & 0,97796 & 0,97956 & 0,98259 & 0,98568 & $-0,32$ & 0,16 & 0,31 & 0,31 \\
65 & 0,97094 & 0,96567 & 0,96541 & 0,9725 & 0,9768 & $-0,54$ & $-0,03$ & 0,73 & 0,44 \\
70 & 0,95421 & 0,95913 & 0,94408 & 0,95518 & 0,96468 & $-0,53$ & $-0,53$ & 1,18 & 0,99 \\
75 & 0,92492 & 0,92115 & 0,91585 & 0,92559 & 0,94302 & $-0,41$ & $-0,58$ & 1,06 & 1,88 \\
80 & 0,87863 & 0,87703 & 0,87714 & 0,88606 & 0,90868 & $-0,18$ & 0,01 & 1,02 & 2,55 \\
85 & 0,80985 & 0,81198 & 0,81905 & 0,83185 & 0,85599 & 0,26 & 0,87 & 1,56 & 2,90 \\
90 & 0,71744 & 0,72079 & 0,7403 & 0,76733 & 0,78686 & 0,47 & 2,71 & 3,65 & 2,55 \\
\hline
\end{tabular}

Tabelle 3: Überlebenswahrscheinlichkeiten nach Altersjahren und prozentuale Veränderungen, weibliche Bevölkerung; Bundesrepublik Deutschland (früheres Bundesgebiet) 1949/51, 1960/62, 1970/72, 1981/83, 1993/95

weibliche Bevölkerung

Voll-

endetes

Altersjahr $x$
Prozentuale Veränderungen

\begin{tabular}{llllll|llllll}
\hline & $1949 / 51$ & $1960 / 62$ & $1970 / 72$ & $1981 / 83$ & $1993 / 95$ & $1949 / 51-1960 / 62$ & $1960 / 62-1970 / 72$ & $1970 / 72-1981 / 83$ & $1981 / 83-1993 / 95$ \\
\hline 0 & 0,95091 & 0,97222 & 0,98016 & 0,99041 & 0,99517 & 2,24 & 0,82 & 1,05 & 0,48 \\
1 & 0,9964 & 0,99799 & 0,99869 & 0,9992 & 0,99949 & 0,16 & 0,07 & 0,05 & 0,03 \\
2 & 0,99785 & 0,99892 & 0,9992 & 0,99949 & 0,99975 & 0,11 & 0,03 & 0,03 & 0,03 \\
5 & 0,99901 & 0,99944 & 0,9995 & 0,99971 & 0,99985 & 0,04 & 0,01 & 0,02 & 0,01 \\
10 & 0,99953 & 0,99972 & 0,99972 & 0,99982 & 0,99988 & 0,02 & 0,0 & 0,01 & 0,01 \\
15 & 0,99932 & 0,9996 & 0,99955 & 0,99966 & 0,9998 & 0,03 & $-0,01$ & 0,01 & 0,01 \\
20 & 0,99885 & 0,99938 & 0,99935 & 0,99954 & 0,99965 & 0,05 & $-0,003$ & 0,02 & 0,01 \\
25 & 0,99865 & 0,99927 & 0,99937 & 0,99952 & 0,99964 & 0,06 & 0,01 & 0,02 & 0,01 \\
30 & 0,99835 & 0,99901 & 0,99923 & 0,99935 & 0,99953 & 0,07 & 0,02 & 0,01 & 0,02 \\
35 & 0,99801 & 0,99862 & 0,99884 & 0,99907 & 0,99929 & 0,06 & 0,02 & 0,02 & 0,02 \\
40 & 0,99745 & 0,99799 & 0,99822 & 0,9986 & 0,99881 & 0,05 & 0,02 & 0,04 & 0,02 \\
45 & 0,99632 & 0,99701 & 0,99718 & 0,99784 & 0,99809 & 0,07 & 0,02 & 0,07 & 0,03 \\
50 & 0,99454 & 0,99555 & 099544 & 0,99657 & 0,99705 & 0,10 & $-0,01$ & 0,11 & 0,05 \\
55 & 0,99187 & 0,99328 & 0,99362 & 0,99484 & 0,99597 & 0,14 & 0,03 & 0,12 & 0,11 \\
60 & 0,98709 & 0,98915 & 0,99012 & 0,99171 & 0,99354 & 0,21 & 0,10 & 0,16 & 0,18 \\
65 & 0,97776 & 0,98138 & 0,98289 & 0,9869 & 0,98949 & 0,37 & 0,15 & 0,41 & 0,26 \\
70 & 0,96089 & 0,96715 & 0,96981 & 0,97781 & 0,98223 & 0,65 & 0,28 & 0,82 & 0,45 \\
75 & 0,93189 & 0,94039 & 0,94571 & 0,95774 & 0,96811 & 0,91 & 0,57 & 1,27 & 1,08 \\
80 & 0,88598 & 0,89669 & 0,90557 & 0,92449 & 0,94297 & 1,21 & 0,99 & 2,09 & 2,00 \\
85 & 0,82638 & 0,83374 & 0,84412 & 0,86941 & 0,8945 & 0,89 & 1,24 & 3,00 & 2,89 \\
90 & 0,74084 & 0,75179 & 0,7658 & 0,79207 & 0,82125 & 1,48 & 1,86 & 3,43 & 3,68 \\
\hline
\end{tabular}



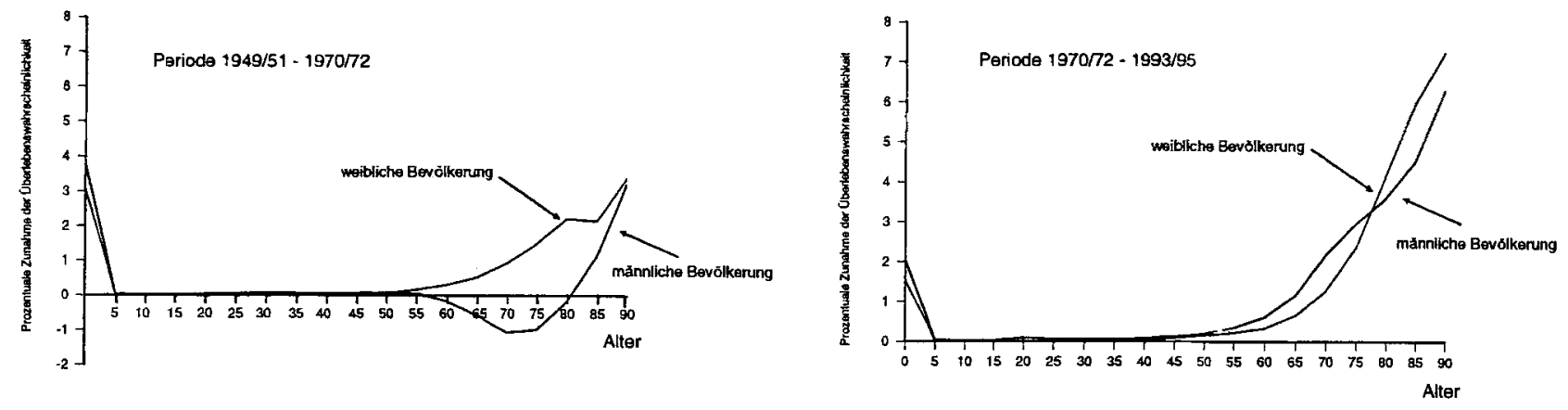

Abbildung 4: Prozentuale Veränderungen der Überlebenswahrscheinlichkeiten nach Altersjahren; Bundesrepublik Deutschland (früheres Bundesgebiet); 1949/51 bis 1970/72, 1970/72 bis 1993/95.

wartung verknüpit, indem vereinfachend »männlich zu sein« ein sozialer Vorteil und »weiblich zu sein« als sozialer Nachteil bewertet wird [17, 18]. Bezüglich der Entwicklung der Lebenserwartung hat aber offensichtlich die weibliche Population in diesem Jahrhundert insgesamt mehr von der Verbesserung der sozialen Lebensqualität (bzw. der »sozialen Lage«) profitiert als die männliche Population. Risiken von Erkrankungen, die bei der Frau mit der Fortpflanzung und den Fortpflanzungsorganen zusammenhängen, sind weitgehend reduziert worden. Günstige soziale Lebensbedingungen führen insbesondere bei Frauen zu einer Zunahme der Lebenserwartung, während in einer menschen- und überlebensfeindlicheren Umwelt Männer eher als Frauen überleben $[19,20]$. Da man von einem mehr oder minder genetisch determinierten konstanten Einfluß auf die Überlebensfähigkeit der Humanpopulation ausgehen kann, ist die geschlechtsdifferente Entwicklung der Lebenserwartung sozialer Natur.

\section{Literatur:}

[1] Schott, J., Bergmann, K. E., und Wiesner, G.: Der Lebensverlängerungsprozeß - ein Vergleich zwischen Ost- und Westdeutschland.
Zeitschr. Bevölkerungswissenschaft 20 (1995) 187-206.

[2] Statistisches Jahrbuch der Bundesrepublik Deutschland. Stuttgart: Metzler-Poeschel Verlag, 1991, 46, und 1997, 47.

[3] Reinschke, K.: Zuverlässigkeit von Systemen. Berlin: Akademie Verlag, 1975, 48.

[4] Meyers, G. C.: Sterblichkeitsrückgang, Lebensverlängerung und Altern der Bevölkerung. Zeitschr. Bevölkerungswissenschaft 10 (1984) 463-475.

[5] Hermalin, A. I.: The Effect of Changes in Mortality Rates on Population Growth and Age Distribution in the United States. Milbank Memorial Fund Quarterly 44 (1966) $451-468$.

[6] Strolnitz, G. J.: Mortality Declines and Age Distribution. Milbank Memorial Fund Quarterly 34 (1956) 178-215.

[7] Radoschewski, M.: Zur Entwicklung der Geschlechtsdifferenzen des Sterberisikos. Beiträge zur Demographie 1 (1977) 223-235.

[8] Hazzard, W. R.: Biological basis of sex differential in longevity. J. A. G. S. 34 (1986) $455-471$.

[9] Hazzard, W. R.: Why do women live longer than men. Postgrad. Med. 85 (1989) 271-283.

[10] Seely, S.: The gender gap: why do women live longer than men? Int. J. Cardiol. 29 (1990) 113-119.

[11] Smith, D. W. E., and Warner, H. R.: Does genotypic sex have a direct effect on longevity? Exp. Gerontology 24 (1989) 277-288.

[12] Waldron, I.: Sex differences in illness incidence, prognosis and mortality: issues and evidence. Soc. Sci. Med. 17 (1983) 1107-1123.

[13] Waldron, I.: Sex differences in human mortality: The role of genetic factors. Soc. Sci. Med. 12 (1983) 321-333.

[14] Wiesner, G., und Haberland, J.: Entwicklung der Suizidsterblichkeit nach der Wiedervereinigung Deutschlands. Bundesgesundhbl. 39,2 (1996) $58-64$.

[15] Weisner, T. S.: Some crosscultural perspectives on becoming female. In: Kopp, C. B. (ed.): Becoming female. New York 1979.

[16] Casper, W., Wiesner, G., und Bergmann, K. E.: Mortalität und Todesursachen in Deutschland. Robert Koch-Institut, RKIHeft 10/1995.

[17] Verbrugge, L. M.: The twain meet: Empirical explanation of sex differences in health and mortality. J. Hlth. Soc. Behav. 30 (1989) 282-304.

[18] Verbrugge, L. M.: Parhways of Health and Death. In: Apple, R. D. (Ed.): Women, Health and Medicine in America. New Brunswick, New Jersey: Rutgers University Press 1990.

[19] Madrigal, L.: Differential sex mortality in a rural nineteenth-century population: Escazu, Costa Rica. Human. Biol. 64 (1992) 199-213.

[20] Riggs, J. E.: Longitudinal gompertzian analysis of adult mortality in the US, 1900-1986. Mechanism Ageing Developm. 54 (1990) 235-247.

Verfasser:

Doz. Dr. Dr. Gerd Wiesner, Edelgard Bittner, Dr. Ingeborg Todzy-Wolff, Robert Koch-Institut

Korrespondenzanschrift:

Doz. Dr. Dr. Gerd Wiesner, Robert Koch-Institut, Postfach 650280, 13302 Berlin 